THE AsTROPHYSICAL JOURNAL, 513:421-427, 1999 March 1

(C) 1999. The American Astronomical Society. All rights reserved. Printed in U.S.A.

\title{
SUBARCSECOND OPTICAL IMAGING OF PROTO-PLANETARY NEBULAE
}

\author{
BRUCE J. HRIVNAK ${ }^{1}$ \\ Department of Physics and Astronomy, Valparaiso University, Valparaiso, IN 46383; bhrivnak@exodus.valpo.edu \\ AND \\ Philip P. Langill, ${ }^{1}$ Kate Y. L. Su, and Sun KwoK ${ }^{1}$ \\ Department of Physics and Astronomy, University of Calgary, Calgary, AB T2N 1N4, Canada; plpl@iras.ucalgary.ca, ysu@iras.ucalgary.ca, \\ kwok@iras.ucalgary.ca \\ Received 1998 April 11; accepted 1998 October 6
}

\begin{abstract}
Subarcsecond (0"75) $V$ and $I$ images have been obtained of 13 new proto-planetary nebulae (PPNs). We find nine of them to be resolved, with sizes up to $3^{\prime \prime}$, and two others to perhaps also be extended. Of the nine, four appear to be elliptical in shape. Thus an aspherical morphology is demonstrated to commonly exist early in the transition between the asymptotic giant branch and planetary nebula phases. The results of this larger observational study, together with the previously published imaging studies, indicate that most PPNs are elliptical (or bipolar) in shape.
\end{abstract}

Subject headings: ISM: Structure — stars: imaging — stars: mass loss — stars: AGB and post-AGB

\section{INTRODUCTION}

Proto-planetary nebulae (PPNs) represent a short-lived, transitional stage in the evolution of intermediate and lowmass stars. This stage is characterized by a detached, expanding circumstellar envelope (CSE) of gas and dust surrounding a central star consisting of a carbon-oxygen degenerate core and a thin hydrogen atmosphere $\left(M_{\text {env }}<\right.$ $10^{-3} M_{\odot}$ ). The expanding envelope represents the mass lost during the preceding asymptotic giant branch (AGB) stage, when mass was lost at an increasingly high rate (up to several times $10^{-4} M_{\odot} \mathrm{yr}^{-1}$ ). The central star is evolving to the left on the H-R diagram, and when its temperature is high enough to photoionize the gas $(T>30,000 \mathrm{~K})$, the object is classified as a planetary nebula (PN). Stellar evolutionary models show that this transitional stage takes only a few thousand years (Blöcker 1995).

Prior to the launch of the $I R A S$ satellite in 1983, only a few PPN candidates were known, in particular AFGL 2688 (Egg Nebula; Ney et al. 1975) and AFGL 618 (Westbrook et al. 1975). Both of these are strong emitters in the infrared, which led to their discoveries. They are also both extended sources, displaying bipolar morphologies in light scattered from their circumstellar envelopes.

We are engaged in a program to discover new PPN candidates, based upon their IRAS colors, and to study them at a variety of wavelengths and with a variety of instruments to understand their properties. PPN candidates show a distinctive double-peaked spectral energy distribution (SED), with one peak, in the visible and near-infrared, representing emission from the reddened photosphere of the central star, and the other peak, in the mid-infrared, representing reradiation by the circumstellar dust. It is known that a large fraction of PNs possess bipolar or axial symmetric shells (Zuckerman \& Aller 1986; Balick 1987; Schwarz, Corradi, \& Melnick 1992). In order to understand the origin of PN morphologies, it would be useful to find out at what stage of PN evolution the nebula becomes asymmetric or bipolar. We address this question in this paper, as we present and

\footnotetext{
${ }^{1}$ Visiting Astronomer at the Canada-France-Hawaii Telescope, operated by the National Research Council of Canada, the Centre National de la Recherche Scientifique of France, and the University of Hawaii.
}

discuss our high-resolution ground-based optical images of a sample of PPNs. The PPNs presented in this paper were first discovered to be the optical counterparts of cool IRAS sources as a result of our search about the IRAS positions with a bolometer at $10 \mu \mathrm{m}$. As a result of this, we are confident of the correct identifications. Identification charts for some of these have been published (Hrivnak, Kwok, \& Boreiko 1985; Hrivnak, Kwok, \& Volk 1988; Hrivnak \& Kwok 1991), while others are in preparation.

\section{OBSERVATIONS AND IMAGE REDUCTION}

High-resolution CCD images were obtained with the $3.6 \mathrm{~m}$ Canada-France-Hawaii Telescope (CFHT) on 1990 August 24, 27, and 28, and 1991 June 4-6 (UT). The DAO/CFHT image-stabilizing High-Resolution Camera (HRCam) was used in the fast guide mode. Details of the design of HRCam are given by McClure et al. (1989). Note that the camera is rotated to position a guide star in the field onto a small hole in the probe mirror, and thus the raw images are not aligned in right ascension and declination. A SAIC $1080 \times 1080$ pixel CCD detector was used with HRCam. Mounted at the CFHT prime focus, each pixel projects an angular size of 0.13 on the sky. Broadband $V$ and $I$ (Mould) filters were used in the observations. The resultant image sizes (FWHM) averaged 0"75. Although this is not as good has been obtained with HRCam at this site, 0"48 (Racine et al. 1991), it is certainly excellent for ground-based imaging and has proved sufficient for us to resolve most of the objects.

The first of the nights in 1990 was a cloudy, nonphotometric half-night (kindly made available to us for imaging work by M. Pierce), and the next 1.5 nights of the 1990 observing run were also nonphotometric owing to cirrus. The last half-night of the 1990 run and all three nights in 1991 were photometric. An observing summary of the imaging program is given in Table 1 , which includes the $I R A S$ identification, the $V$ magnitude, the exposure times in each filter, the image seeing based upon the FWHM of brighter stars in the image, the object size (FWHM) of our program objects, sky conditions (photometric or nonphotometric), and the observation dates. Comparison of the object size with the image seeing was used to indicate 
TABLE 1

OBSERVING LOG

\begin{tabular}{|c|c|c|c|c|c|c|c|c|c|}
\hline \multirow[b]{2}{*}{$I R A S$} & \multirow[b]{2}{*}{$V^{\mathrm{a}}$} & \multicolumn{2}{|c|}{ EXPOSURE TIME (s) } & \multicolumn{2}{|c|}{ IMAGE SEEING (FWHM, arcsec) } & \multicolumn{2}{|c|}{ ОвJеCт SIZe (FWHM, arcsec) } & \multirow[b]{2}{*}{ SKY CONDITIONS ${ }^{\mathrm{b}}$} & \multirow[b]{2}{*}{ DATE } \\
\hline & & $V$ & $I$ & $V$ & $I$ & $V$ & $I$ & & \\
\hline $16559-2957 \ldots \ldots$ & 13.2 & 60 & 20 & $0.62^{\mathrm{c}}$ & $0.63^{\mathrm{c}}$ & $0.65^{\mathrm{c}}$ & $0.64^{\mathrm{c}}$ & $\mathbf{P}$ & 1991 Jun 4 \\
\hline $17106-3046 \ldots \ldots$ & 15.8 & 300 & 60 & 0.98 & 0.84 & 1.23 & 1.00 & NP & 1990 Aug 27 \\
\hline $17245-3951 \ldots \ldots$ & 15.5 & 600 & 200 & 1.04 & $(0.83)^{\mathrm{c}}$ & 1.24 & $(1.10)^{\mathrm{c}}$ & $\mathbf{P}$ & 1991 Jun 5 \\
\hline $18095+2704 \ldots \ldots$ & 10.4 & 5 & 1 & 0.85 & 0.7 & 0.90 & 0.7 & NP & 1990 Aug 27 \\
\hline $19374+2359 \ldots \ldots$ & 18.1 & 600 & 240 & 0.67 & 0.64 & 1.15 & 1.00 & $P$ & 1991 Jun 6 \\
\hline $19475+3119 \ldots \ldots$ & 9.4 & $\ldots$ & 60 & $\ldots$ & $\sim 1.00^{\mathrm{c}, \mathrm{d}}$ & $\ldots$ & $\sim 1.00^{\mathrm{c}, \mathrm{d}}$ & NP & 1990 Aug 24 \\
\hline $19477+2401 \ldots \ldots$ & 22.2 & 1800 & 600 & 0.68 & 0.66 & $\ldots$ & 0.77 & $\mathrm{P}$ & 1991 Jun 5 \\
\hline $20000+3239 \ldots \ldots$ & 13.3 & 80 & 4 & 0.71 & 0.61 & 0.76 & 0.69 & NP & 1990 Aug 27 \\
\hline $20028+3910 \ldots \ldots$ & 17.8 & $\ldots$ & $100^{\mathrm{e}}$ & $\ldots$ & $0.54^{\mathrm{e}}$ & $\ldots$ & $0.83^{\mathrm{e}}$ & NP & 1990 Aug 24 \\
\hline $22574+6609 \ldots \ldots$ & 24.0 & 900 & 1500 & 0.81 & 0.76 & 0.92 & 0.87 & NP & 1990 Aug 27 \\
\hline $23304+6147 \ldots \ldots$ & 13.1 & 30 & 5 & $0.68^{\mathrm{c}}$ & $0.63^{\mathrm{c}}$ & $0.84^{\mathrm{c}}$ & $0.75^{\mathrm{c}}$ & $\mathrm{P}$ & 1990 Aug 28 \\
\hline $00210+6221 \ldots \ldots$ & 16.5 & 720 & 360 & 0.95 & $0.94^{\mathrm{c}}$ & 1.00 & $0.96^{\mathrm{c}}$ & NP & 1990 Aug 27 \\
\hline $01174+6110 \ldots \ldots$ & 18.3 & 450 & 420 & 0.72 & 0.72 & 0.84 & 0.78 & $P$ & 1990 Aug 28 \\
\hline
\end{tabular}

${ }^{a} V$ magnitudes from our unpublished photometry or from references cited in the paper.

${ }^{\mathrm{b}}$ Sky conditions are listed as either photometric (P) or nonphotometric (NP).

c Asymmetric profiles, or FWHM varies across field of image.

${ }^{d}$ Observed with the camera aperture reduced to an effective telescope aperture of $1.2 \mathrm{~m}$.

${ }^{\text {e }}$ Observed with a broadband $R$ filter.

extended sources. In some cases we have made multiple exposures, occasionally of different exposure times. The ones listed and used here are those with the best image quality.

Nightly flat-field images of the twilight sky and bias measurements were made, and the images of the stellar fields were corrected for these, using IRAF. ${ }^{2}$ The photometric results will be discussed elsewhere and will include finding charts for the objects for which these have not been published. Here we will focus our discussion on the highresolution images of the objects and on the study of extended emission due to scattered light from the circumstellar shell.

\section{ANALYSIS OF EXTENDED IMAGES}

It is apparent that some of our program objects are extended in angular size, as is indicated by a comparison of their FWHM with that of stars in the field (see Table 1). This is what one would anticipate if light were scattered by the dust in a resolved shell of material around the central star.

To examine the size and shape of the extended emission, we performed two different, independent analyses of the extended images. The first was to remove the light of the central star through a point-spread function subtraction. This would permit us to investigate the size and shape of the residual light due to the extended emission. The second method was to do a deconvolution of the PPN image by the point-spread function to improve the resolution. We will discuss in more detail the two methods and the results of these analyses.

\subsection{Point-Spread Function Subtraction}

This method involved an attempt to isolate the scattered light by removing the light from the central star through

\footnotetext{
2 The Image Reduction and Analysis Facility (IRAF) is distributed by the National Optical Astronomy Observatories, which is operated by the Association of Universities for Research in Astronomy, Inc., under contract to the National Science Foundation.
}

subtraction of a point-spread function (PSF). A PSF is essentially an average stellar profile determined by combining the stellar profiles of a number of field stars from the same image. The program DAOPHOT within IRAF was used in this analysis (Stetson 1987; Stetson, Davis, \& Crabtree 1990).

We proceeded in the following manner. We first formed the PSF for an image, then scaled it to the integrated brightness of the PPN, and finally subtracted it. Since the FWHM of the profile of an extended object will be larger than that of the PSF, the resulting residual will be a "hole" of "negative light" in the center of a circular or elliptical distribution of residual light. (In the elliptical case, it will cause the resulting light distribution to appear bipolar.) The "negative hole" arises because we initially assumed that all of the light of the extended source was due to the central star; certainly this is incorrect, since some is from the extended emission. The next step was to reduce the amount of light attributed to the central star by rescaling the PSF subtracted until the hole in the center of the residual light is no longer negative but has a value of zero (above the sky). This corresponds to assuming that no light is scattered in the forward direction. On the assumption that some light is indeed scattered into the forward direction, we proceeded to further reduce the amount of light attributed to the central star and thus fill in the hole in the light distribution. Thus, the PSF was scaled such that the resulting dust shell emission no longer shows signs of a central depression. The amount by which the PSF was eventually scaled to give this smooth-topped scattered light distribution also depended on the alignment between the PSF and the initial light distribution. Thus the PSF subtraction was a threedimensional, trial-and-error process. For more details see Langill (1994).

The final result of this PSF subtraction procedure does not guarantee that the direct light of the central star of the program object has been correctly subtracted. The effect of the uncertainty in the scaling of the PSF on the final, PSFsubtracted image is obviously greatest for the inner region. However, we find that the scaling of the light attributed to 
the central star has little effect on the outer contours with regard to their size or shape. The angular size and shape of the faint and extended dust emission remain essentially unchanged for most of the objects even if the PSF is further reduced by as much as $50 \%$. Thus we have confidence that the overall shape of the resulting contour plot accurately represents the general morphology of the dust shell.

\subsection{Image Deconvolution}

In this method, we used the maximum entropy method within STSDAS to deconvolve the PSF with the extended image of the PPN. Due to the nature of the process of deconvolving the PSF with the image, it is even more crucial that we have a good PSF in this method than in the PSF-subtraction method. In some cases this could not be accomplished owing to variations in the PSF across the field or, in the case of very bright PPNs, owing to the lack of good field stars. In the cases with a good PSF, we then deconvolved a subset of the image around the PPN. We set the deconvolution to proceed for at least 100 iterations, then stopped at a convergence. When successfully applied, this method produced much sharper images of the stars in the field and allowed for a more precise measurement of the size of the extended emission.

\subsection{Comparison of the Methods}

We obtained the same general results with both the PSFsubtraction and the deconvolution methods, in the sense that the resultant PPN images had the same shapes and orientations. However, the deconvolution method produced a sharper image of the extended emission from which to judge the shape and measure the size. Thus the quantitative results will come from this method.

One could also have considered a combination of the two methods, first deconvolving the PPN image and then subtracting a new PSF based upon the deconvolved images of the field stars. However, we have not done this, for we think that the deconvolved images show the extended emission clearly enough. Also, the PSFs formed often are not of sufficient quality to warrant a second manipulation of the data with them.

\subsection{Discussion of Results for Individual IRAS Objects}

16559-2957.- This object has a bright optical counterpart, $V=13.2$. This source has been correctly identified and observed photometrically by $\mathrm{Hu}$ et al. (1993), who found a similar magnitude. However, they state that it is associated with a compact reflection nebula of diameter $\sim 12^{\prime \prime}$. We see no indication of an associated nebula on our images. The stellar profiles are noncircular and vary noticeably across the field, making the FWHM difficult to measure and leading to a poor PSF. The deconvolution consequently produced poor results. Given these uncertainties, it does not appear that the IRAS source is extended.

17106-3046.-This object clearly appears to be extended, based upon a comparison of its FWHM with that of stars in the field (see Table 1). The PSF subtraction for both the $V$ and $I$ images revealed extended emission, with an outer halo that is round and inner intensity contours that are somewhat elliptical, especially in $V$. The same morphology is seen in the deconvolved images. The angular diameter is 2"9 in $V$. The inner elliptical shape seen in the $V$ image extends northwest to southeast. We show in Figure
$1 a$ a false-color picture of the deconvolved $V$ image to give the overall impression of the morphology and, in Figure $2 a$, a isophotal contour plot on an expanded scale to show more detail of the inner contours.

17245 - 3951.- This object also appears to be extended from its FWHM. In the $I$ image, the PSF is not symmetric and varies systematically in FWHM across the entire image; therefore we have not used it in the analysis. The PSF-subtracted $V$ image of the IRAS source is clearly elliptical. The deconvolved $V$ image gave good results, with the nebula showing an angular size of 1".6 $\times 0$ 0".9 and aligned north-south, with the brightness peak north of center in the image. This is displayed in Figures $1 b$ and $2 b$.

$18095+2704$. - This object is identified with a finding chart and discussed by Hrivnak et al. (1988). There is a suggestion from the FWHM of the $V$ image that the source is slightly extended as compared with the average of the field stars. However the FWHM is found to vary across the field, making it difficult to form a good PSF for use in the image. An additional complication is that the object is so much brighter $(V=10.4)$ than all of the other stars in the field. Thus we were not able to do a quantitative analysis of the possible extended emission.

$19374+2359$. - This object is clearly extended, as indicated by its FWHM. A map of this field identifying the IRAS source has been published (Hrivnak et al. 1985). The PPN image appears to have an approximately round nebula in both analyses. We determine a diameter of 2".6 in $V$ from the deconvolved image of the PPN, which is shown in Figures $1 c$ and $2 c$.

$19475+3119$. - This is a very bright object, $V=9.4$ (HD 331319, BD +31 ${ }^{\circ} 3797$, LS II $+31^{\circ} 9$ ). In an effort to improve the seeing and reduce the brightness, we observed with the camera aperture reduced to an effective telescope aperture of $1.2 \mathrm{~m}$. An $I$ image was taken under cirrus cloud conditions. The resulting stellar profiles were highly nonsymmetrical, making a search for extended emission by comparing image size with image seeing highly uncertain. There is no evidence that the object is extended.

$19477+2401$. - The faint optical counterpart of this source was previously identified and an identification chart published (Hrivnak et al. 1998). This source is both very faint in $V$ and extremely red. In the long $V$-band exposure, the PPN was barely detectable, and only the $I$ image was usable in the analyses. The PPN image was clearly extended and slightly elliptical in both analyses, and we measure a maximum size of $\sim 1$ ". 0 , oriented approximately northeast by southwest.

$20000+3239$. - This object is very bright and red, with $V=13.3$ and $V-I=3.0 \mathrm{mag}$ (Kwok, Hrivnak, \& Geballe 1995), and is much brighter than any of the other stars in the field, especially in the $I$ band. Exposures through cirrus clouds were short, as listed in Table 1 . The $V$ image is less noisy and permitted a better PSF to be constructed. The PPN appears to be slightly extended and round in shape, with a size on the deconvolved image of 1".6 in diameter.

$20028+3910$. - This source is faint and red, $V=17.8$ and $V-I=2.5$. It clearly appears to be extended based upon its FWHM. It was observed on a nonphotometric night through a broadband $R$ filter. The PSF formed was asymmetric. The result of the analyses indicates that the image is elliptical with a size of $2 . .2 \times 2.0$ and an approximately north-south orientation. This is shown in Figure 1d, where one can see the elliptical shape and also the poor PSF of a 


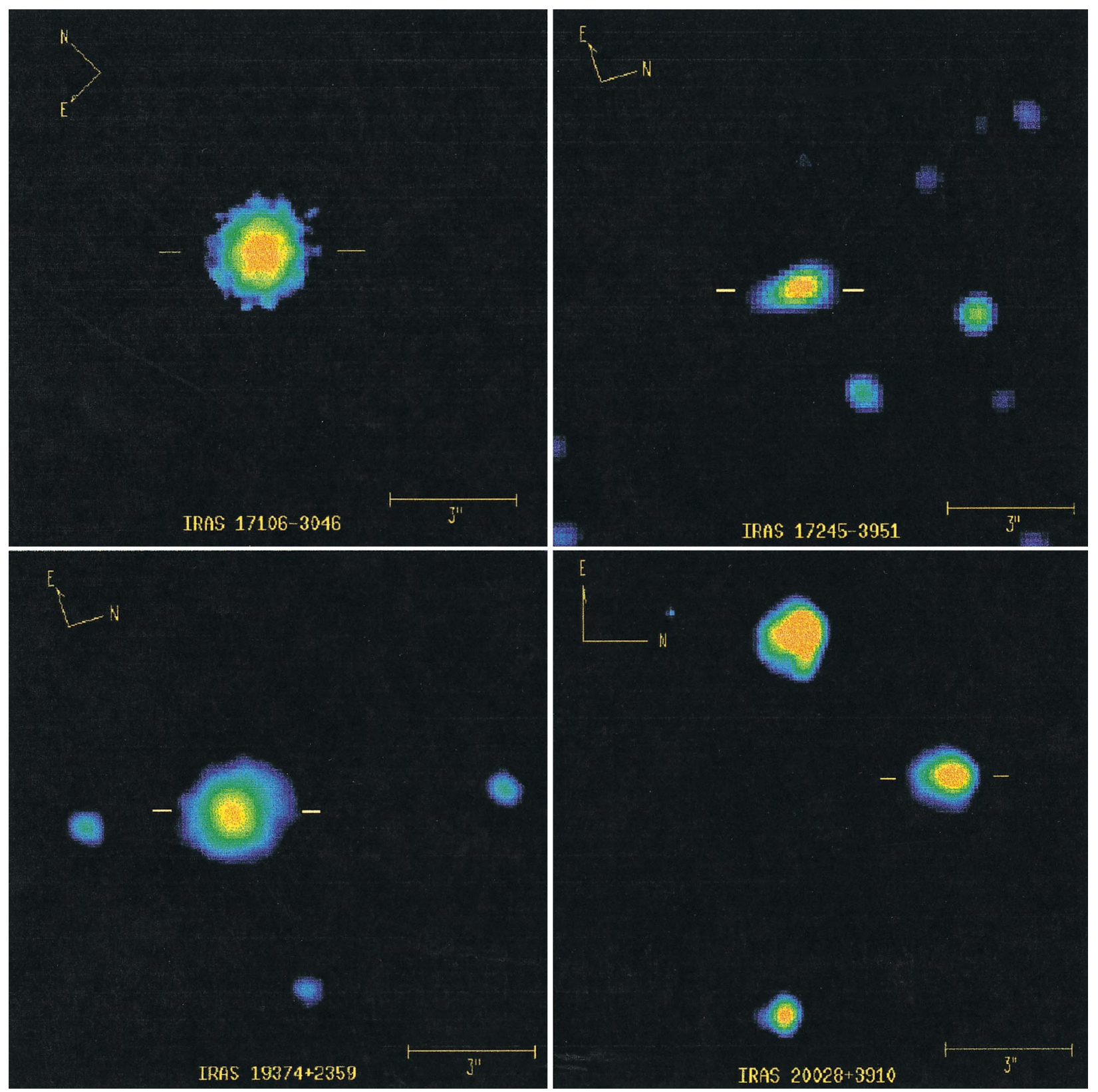

FIG. 1.- False-color pictures of four resolved PPNs, showing their overall morphology. These are the deconvolved images and are plotted on a linear scale. Upper left: IRAS 17106-3046; elliptical inner brightness and round outer brightness. Upper right: IRAS 17245-3951; elliptical. Lower left: IRAS 19374+2359; round. Lower right: IRAS 20028+3910; elliptical, but note the asymmetrical shapes of the field stars. The orientation and scale are noted for each.

bright field star. Figure $2 d$ shows clearly the elliptical shape of the inner contours.

$22574+6609$. - This source is very faint $(V=24)$ and red, and a finding chart and discussion of the object are presented by Hrivnak \& Kwok (1991). It appears to be extended in both the $V$ and $I$ images, judging by its FWHM. We investigated the brighter $I$ image of the PPN and find it to be approximately round, although with perhaps a suggestion of slight elongation in the north-south direction, and to have a diameter of $\lesssim 1.0$.

$23304+6147$. - This object is very bright and red, $V=13.1$ and $V-I=2.6$, and a finding chart and discussion of this object are also found in Hrivnak \& Kwok (1991). As was the case with $20000+3239$, the exposure times were short, and this resulted in a rather noisy back- ground, especially in the $I$ band. The images of all stars in the field are somewhat asymmetric, particularly in the $I$ image. Because of this, we were not able to form a good PSF. It is clear from the size of the FWHM that the PPN is extended. Our analyses suggest that it is approximately round, with a size on the deconvolved image of $\sim 2$ ".0 diameter.

$00210+6211$. - An identification map of this field was published by Hrivnak et al. (1985). For this object, its FWHM seems to be slightly larger than that of the stars in the field (see Table 1), suggesting that the source may be extended. However, the analysis is complicated by a PSF that is variable across the $I$ image. We simply conclude that it is perhaps extended, but this should be confirmed with new, higher resolution images. 

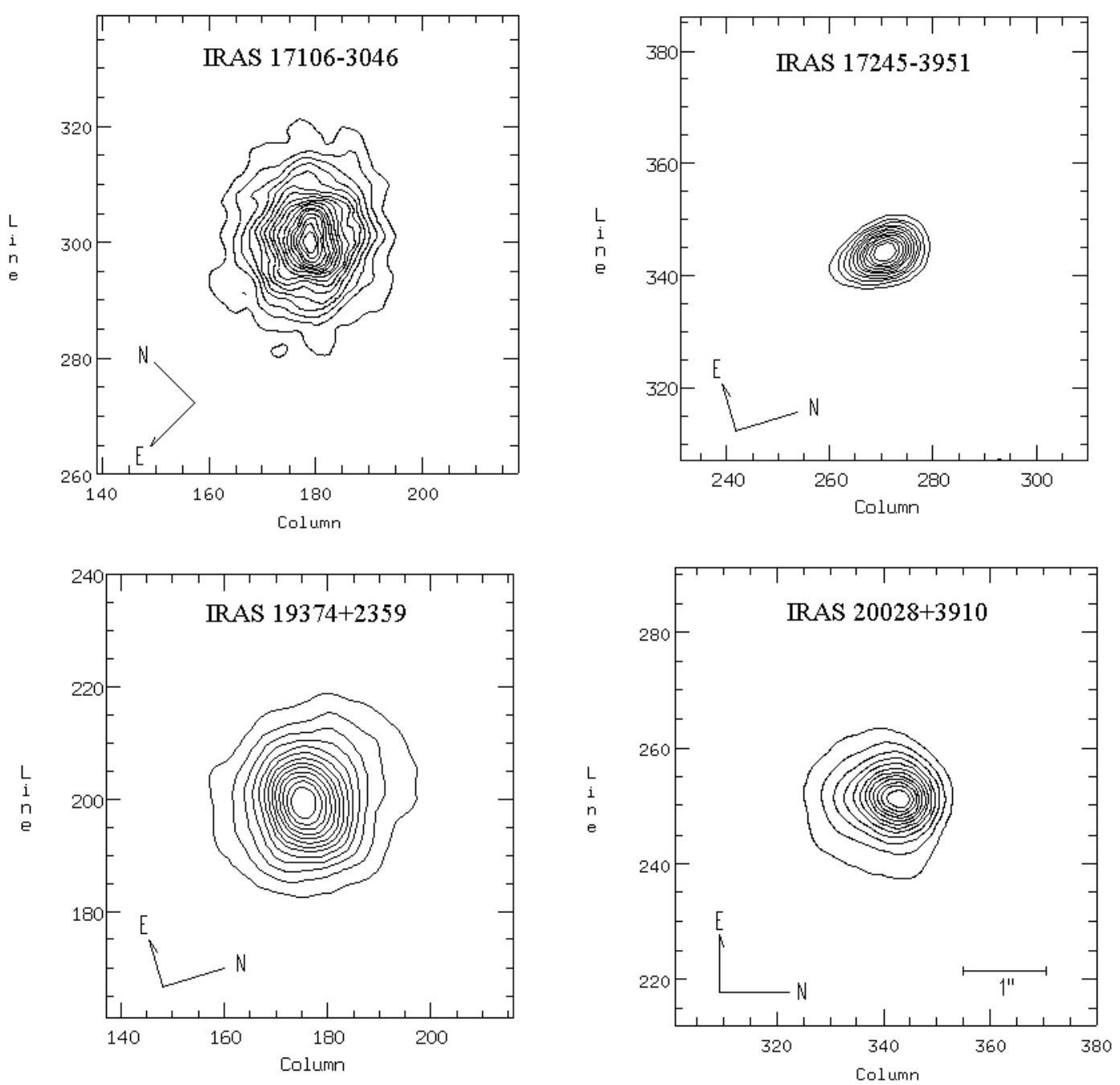

FIG. 2.- Isophotal contour maps of the same four PPNs as in Fig. 1, but on an expanded scale. Here the morphologies are more clear, especially the inner elliptical contours of IRAS 17106-3046. Note also that the brightness peak of IRAS 17245-3951 is off-center. The lowest contour is plotted at $10 \%$ of the peak brightness above the sky, and the contours continue at intervals of $10 \%$ up to the $90 \%$ level. The orientation is noted for each, and the images are all on the same scale, which is indicated on the lower right.

$01174+6110$. - There are only a few stars to use to form a PSF in the $V$ image, where the PPN is much brighter than the stars in the field. In the $I$ image, a good PSF could not be formed. We thus could not do a good analysis with either method. We simply conclude from the FWHM that the object is probably extended, but cannot measure a size accurately.

\section{DISCUSSION}

In this study, we have used two different methods to reveal faint scattered emission from the extended CSEs of PPNs. This is the largest sample of PPNs imaged to date, and it approximately doubles the number known to be resolved. These results are summarized in Table 2. In every case where a good PSF could be constructed from surrounding field stars, extended emission from a CSE was found or suggested.

In half of the clearly resolved cases, four out of eight, the extended emission appears elliptical. Thus even in this early stage of post-AGB evolution, the morphology of the nebulae is often aspherical. Even for those PPNs whose CSEs appear round, it is possible that some are actually aspherical but are either (1) small compared to the PSF, so that we cannot distinguish the ellipticity (IRAS $22574+6609$ ), or (2) oriented with their major axis aligned near the direction of the line of sight, so that they appear round at this resolution. Thus the number of PPNs with elliptical (or the more extreme bipolar) CSEs might be higher than that shown in Table 2. This is clear evidence that the seeds of aspherical PN morphologies take root long before photoionization occurs.

It would be difficult to distinguish between elliptical and bipolar morphologies for objects with the small angular extent of those in this study. The situation is more clear for the two bipolar nebulae IRAS 17150-3224 and IRAS $17441-2411$, recently discussed by Kwok et al. (1996). They are large, $15^{\prime \prime} \times 8^{\prime \prime}$ and $11^{\prime \prime} \times 7^{\prime \prime}$, respectively, have wellresolved lobes, and are observed nearly edge-on. The objects in the present study are smaller, and it is possible that some of them are also bipolar, but with lobes that are unresolved. This would take higher resolution imaging, such as is obtainable with the Hubble Space Telescope (HST).

Circumstantial evidence for aspherical CSEs had been inferred in earlier work. From a study of three PPNs with similar mid-infrared fluxes but different visible and near- 
TABLE 2

SUMMARY OF EXTENDED EMISSION

\begin{tabular}{clcccl}
\hline \hline \multicolumn{1}{c}{ IRAS } & Shape & $\begin{array}{c}\text { Size } \\
(\mathrm{arcsec})\end{array}$ & Filter & $\begin{array}{c}\text { Chemistry } \\
(\mathrm{C} \text { or O) }\end{array}$ & \multicolumn{1}{c}{ Comments on Images } \\
\hline $16559-2957 \ldots \ldots$ & \multicolumn{1}{c}{$\ldots$} & $\ldots$ & $\ldots$ & O & Not extended \\
$17106-3046 \ldots \ldots$ & Elliptical/Round & 2.9 & $V$ & O & Elliptical inner, P.A.: NW-SE \\
$17245-3951 \ldots \ldots$ & Elliptical & $1.6 \times 0.9$ & $V$ & $?$ & P.A.: N-S \\
$18095+2704 \ldots \ldots$ & $\ldots$ & $\ldots$ & $\ldots$ & O & Perhaps extended \\
$19374+2359 \ldots \ldots$ & Round & 2.6 & $V$ & $?$ & \\
$19475+3119 \ldots \ldots$ & $\ldots$ & $\ldots$ & $\ldots$ & $?$ & Not extended \\
$19477+2401 \ldots \ldots$ & Elliptical: & $<1$ & $I$ & $?$ & P.A.: NE-SW \\
$20000+3239 \ldots \ldots$ & Round & $\sim 1.6$ & $V$ & C & Probably extended \\
$20028+3910 \ldots \ldots$ & Elliptical & $2.2 \times 2.0$ & $R$ & $?$ & P.A.: N-S \\
$22574+6609 \ldots \ldots$ & Round: & $<1$ & $I$ & C & Perhaps elliptical \\
$23304+6147 \ldots \ldots$. & Round & $\sim 2.0$ & $V$ & C & \\
$00210+6221 \ldots \ldots$ & & $\ldots$ & $\ldots$ & $?$ & Perhaps extended \\
$01174+6110 \ldots \ldots$ & ? & $\ldots$ & $\ldots$ & O & Probably extended \\
\hline
\end{tabular}

infrared fluxes, Hrivnak \& Kwok (1991) suggested that the three objects might be represented by the same bipolar model viewed from different orientation angles. Two of these three PPNs are observed in the present imaging study. For IRAS $22574+6609$, which is faint in visible light, they suggested that direct light from the star is being obscured by a dust torus, that the polar axis is more nearly in the plane of the sky, and that it is the most likely to appear as bipolar. For IRAS $23304+6147$, which is bright in visible light, they suggest that one is viewing the object more nearly pole-on. These suggestions are not inconsistent with the high-resolution optical images presented here: IRAS $22574+6609$ appears round, but with a suggestion of ellipticity, and IRAS $23304+6147$ appears round. Higher resolution images of IRAS $22574+6147$ would be needed to determine whether the object is bipolar. According to this model, the third object, IRAS $04296+3429$, would also appear nearly circular.

Mid-infrared imaging has recently been carried out of several new PPNs, in addition to the well-known bipolar cases AFGL 2688 and AFGL 618. At these wavelengths, the CSE may be optically thin, which would permit the direct imaging of the dust. Two oxygen-rich PPN have been imaged and resolved, one of which shows a central torus and circular outer envelope (HD 161796 = IRAS $17436+5003$, size $=3^{\prime \prime}$; Skinner et al. 1994) and one of which is circular (HD 179821 = IRAS 19114+0002, size $=5^{\prime \prime}$; Hawkins et al. 1995). Four carbon-rich sources that display $21 \mu \mathrm{m}$ emission (Kwok, Volk, \& Hrivnak 1989) have been imaged, and three resolved. All three of those resolved appear elliptical, and in one the removal of the light of the central star results in a torus of emission (Meixner et al. 1997). Unfortunately, none of the objects imaged in the mid-infrared is in common with our sample of visible PPNs. This is due primarily to the fact that we observed fainter PPNs in the visible to avoid saturation of the $C C D$, while in the mid-infrared, where saturation was not a problem, the brighter ones were observed. However, the results of these mid-infrared studies are similar to ours in that they also find the majority of these objects to display aspherical morphologies.

An additional means to search for aspherical CSEs in PPNs is by polarization measurements, since scattering of light by dust particles with a spherical geometry will produce no net polarization, while an aspherical geometry will. Three of the objects in this study have polarization measurements by Trammell, Dinerstein, \& Goodrich (1994). They found IRAS $18095+2704$ to possess large, intrinsic polarization; we find the object to perhaps be extended but have no information on its shape. They find IRAS $20000+3239$ and IRAS $23304+6147$ to have no intrinsic polarization, which is in agreement with the round shapes that we find.

Thus the results of this study of visible imaging, combined with previous visible imaging and the recent midinfrared imaging, indicate that the majority of spatially resolved PPNs (12 out of 18) possess aspherical CSEs. Therefore the change from the spherical envelope of an AGB star to the aspherical shell of a PN is clearly evident early in the transitional PPN stage.

All of the objects in this study display bimodal SEDs, which indicates that they possess detached CSEs, although in some cases the peak in the visible and near-infrared is significantly weaker than in the mid-infrared peak. Spectral types have been obtained for the five brightest sources, and all have the spectra of $F$ or $G$ supergiants. Expanding circumstellar molecular envelopes have been observed around most of these sources (Likkel et al. 1991; Omont et al. 1993). Thus these objects display the properties expected of objects in the transitional PPN stage.

A few of the sources possess the $10 \mu \mathrm{m}$ silicate absorption feature in their mid-infrared spectra (IRAS 16559-2957 and perhaps IRAS $01174+6110$ ), and are presumably oxygen rich, as is IRAS $18095+2704(\mathrm{OH} 53.8+20.2)$. $\mathrm{OH}$ emission has been reported for IRAS 17106-3046 (Silva et al. 1993), which indicates that it is also oxygen rich, as well as for the other oxygen-rich sources listed above. Three are clearly carbon rich (IRAS 20000+3239, IRAS $22574+6609$, IRAS $23304+6147$ ), and these show the 21 $\mu \mathrm{m}$ emission feature. For the others, the chemistry of the CSE is not yet determined. The sample thus contains a mixture of oxygen-rich and carbon-rich sources. We find no obvious correlation between the shape and the chemistry of the CSE.

Now that the extended nature of these sources and some information on their shapes have been found, follow-up observations at higher resolution are desirable to determine their detailed structures. Such studies have recently been carried out with the $H S T$ for the larger and better studied PPNs AFGL 2688 (the Egg Nebula; Sahai et al. 1998a, 
1998b), AFGL 915 (the Red Rectangle; Bond et al. 1997), IRAS 17150 - 3224 (the Cotton Candy Nebula; Kwok, Su, \& Hrivnak 1998), and IRAS 17441-2411 (the Silkworm Nebula; Su et al. 1998). For the brightest of our new sources, which were difficult for us to observe, a coronagraph could be used to occult the bright central star and allow the faint, extended emission to be detected. Groundbased adaptive optics would also be useful, especially in the near-infrared. Since many of these objects possess a relatively bright point source in the center, a high-speed shiftand-add system in the near-infrared or visible would have the potential to bring out the details in their faint circumstellar shells.

This more detailed information on the morphology of the CSE will lead to more accurate modeling of the properties of the CSEs of these systems. Initial models assumed spherical symmetry and were constructed to fit the SED only. With morphological information, especially at multiple wavelengths, two-dimensional models will now be required to fit both the SED and the morphology.

\section{CONCLUSIONS}

We have obtained subarcsecond imaging observations for a sample of 13 IRAS sources that we have identified as
PPN candidates. In spite of their small sizes, we have found nine of the 13 to be extended, and four among these to show evidence for aspherical nebulosity. This suggests that the shaping of PN begins early, even in the PPN stage. Higher spatial resolution imaging, e.g., by the $H S T$, should provide a more detailed picture of their morphology and help answer the question of the origin of the bipolar structure in PNs.

We thank R. Racine for his help in the use of HRCam, M. Pierce for sharing some observing time, and $\mathrm{H}$. Harris for first suggesting to us the extended nature of IRAS $19374+2359$ and IRAS $20028+3910$. P. Stetson kindly reviewed a few of our images for the presence of extended emission and looked over the manuscript. The comment of the referee to sharpen the presentation and the urging to try image deconvolution have led to a better paper. This research was supported in part by grants to B. J. H. from the National Science Foundation (AST-9018032, AST9315107) and the NASA Astrophysics Data Program (NAG 5-1223) and in part by grants to S. K. from the Natural Sciences and Engineering Research Council of Canada. This research made use of the SIMBAD database, operated at CDS, Strasbourg, France.
Balick, B. 1987, AJ, 94, 671

Blöcker, T. 1995, A\&A, 299, 755

Bond, H. E., Fullton, L. K., Schaefer, K. G., Ciardullo, R., \& Sipior, M. 1997, in IAU Symp. 180, Planetary Nebulae, ed. H. J. Habing \& H. J. G. L. M. Lamers (Dordrecht: Kluwer), 211

Hawkins, G. W., Skinner, C. J., Meixner, M. M., Jernigan, J. G., Arens,

J. F., Keto, E., \& Graham, J. R. 1995, ApJ, 452, 314

Hrivnak, B. J., \& Kwok, S. 1991, ApJ, 368, 564

Hrivnak, B. J., Kwok, S., \& Boreiko, R. T. 1985, ApJ, 294, L113

Hrivnak, B. J., Kwok, S., \& Volk, K. M. 1988, ApJ, 331, 832

Hu, J. Y., Slijkhuis, S., de Jong, T., \& Jiang, B. W. 1993, A\&AS, 100, 413

Kwok, S., Hrivnak, B. J., \& Geballe, T. R. 1995, ApJ, 454, 394

Kwok, S., Hrivnak, B. J., Zhang, C. Y., \& Langill, P. P. 1996, ApJ, 472, 287

Kwok, S., Su, K. Y. L., \& Hrivnak, B. J. 1998, ApJ, 501, L117

Kwok, S., Volk, K. M., \& Hrivnak, B. J. 1989, ApJ, 345, L51

Langill, P. P. 1994, Ph.D. thesis, Univ. Calgary

Likkel, L., Forveille, T., Omont, A., \& Morris, M. 1991, A\&A, 246, 153

McClure, R. D., et al. 1989, PASP, 101, 1156

Meixner, M. M., Skinner, C. J., Graham, J. R., Keto, E., Jernigan, J. G., \& Arens, J. F. 1997, ApJ, 482, 897

\section{REFERENCES}

Ney, E. P., Merrill, K. M., Becklin, E. E., Neugebauer, G., \& WynnWilliams, C. G. 1975, ApJ, 198, L129

Omont, A., Loup, C., Forveille, T., te Lintel Hekkert, P., Habing, H., \& Sivagnanam, P. 1993, A\&A, 267, 515

Racine, R., Salmon, D., Cowley, D., \& Sovka, J. 1991, PASP, 103, 1020

Sahai, R., et al. 1998a, ApJ, 493, 301

. 1998b, ApJ, 492, L163

Schwarz, H. E., Corradi, R. L. M., \& Melnick, J. 1992, A\&AS, 96, 23

Silva, A. M., Azcarate, I. N., Poppel, W. G. L., \& Likkel, L. 1993, A\&A, 275,510

Skinner, C. J., Meixner, M. M., Hawkins, G. W., Keto, E., Jernigan, J. G., \& Arens, J. F. 1994, ApJ, 423, L135

Stetson, P. B. 1987, PASP, 99, 191

Stetson, P. B., Davis, L., \& Crabtree, D. 1990, in ASP Conf. Ser. 8, CCDs in Astronomy, ed. G. H. Jacoby (San Francisco: ASP), 289

Su, K. Y. L., Volk, K., Kwok, S., \& Hrivnak, B. J. 1998, ApJ, 508, 744

Trammell, S. R., Dinerstein, H. L., \& Goodrich, R. W. 1994, AJ, 108, 984

Westbrook, W. E., Becklin, E. E., Merrill, K. M., Neugebauer, G., Schmidt, M., Willner, S. P., \& Wynn-Williams, C. G. 1975, ApJ, 202, 407

Zuckerman, B., \& Aller, L. H. 1986, ApJ, 301, 772 